\title{
INVESTIGACIONES
}

\section{Percepción del profesorado español sobre el alumnado con altas capacidades}

\author{
Spanish teachers' perception of gifted students \\ Espanhol percepção dos professores sobre os alunos com altas capacidades
}

\author{
Alba García-Barrera, ${ }^{a}$ Patricia de la Flor ${ }^{b}$
}

${ }^{a}$ Universidad a Distancia de Madrid (UDIMA)

Telf.: (34) 9118969 94. Correo electrónico: alba.garcia@udima.es

bUniversidad a Distancia de Madrid (UDIMA)

Telf.: (34) 9118969 94. Correo electrónico: patricia_delaflor@hotmail.com

\begin{abstract}
RESUMEN
Actualmente se estima que en España entre un $95 \%$ y un $99 \%$ de los estudiantes con altas capacidades intelectuales están sin identificar. El presente trabajo, sensible a esta realidad, constituye una aproximación a las percepciones que el profesorado de Secundaria manifiesta respecto a este alumnado. Para ello, se diseñó un cuestionario que completaron 637 docentes de Educación Secundaria de todo el territorio español, de los cuales 176 afirmaron poseer formación específica respecto a estos estudiantes. Los datos obtenidos revelan que una parte considerable de los participantes posee escasos conocimientos sobre este alumnado y sigue incurriendo en los típicos mitos acerca de ellos, resultando alarmante que incluso los profesores con formación específica sobre AACI muestren dichas carencias y confusiones. Todo ello dificulta la correcta detección y atención educativa de estos alumnos, por lo que convendría averiguar en qué está fallando la formación docente, a fin de reforzarla desde su inicio.
\end{abstract}

Palabras clave: alumnos con altas capacidades intelectuales, educación secundaria, formación del profesorado, educación inclusiva, medidas educativas.

\section{ABSTRACT}

Nowadyas, it is estimated that between $95 \%$ and $99 \%$ of gifted students are unidentified. This paper, sensitive to this reality, constitutes an approach to perceptions that secondary school teachers manifested regarding these students. To do this, we designed a questionnaire which was completed by 637 teachers of Secondary Education of the whole Spanish territory, of which 176 have stated specific training regarding these students. These results show that a significant part of participants has limited knowledge about these students and continues to follow common myths regarding these students. It results alarming that even teachers with specific training on gifted students show those shortcomings and confusion. This complicates the correct detection and educational attention to these students, so it would be desirable to find out what is wrong with this teacher training, in order to strengthen it since its inception.

Key words: gifted students, secondary education, teacher training, inclusive education, educational measures. 


\section{INTRODUCCIÓN}

Es indudable hoy en día la importancia que tiene la formación y preparación del profesorado para atender la diversidad existente en sus aulas. Entre esta diversidad se encuentra el alumnado con altas capacidades intelectuales, a los que desde este momento, para simplificar la notación, denominaremos AACI.

Según Miguel y Moya (2012), el concepto de alumno con altas capacidades intelectuales es difícil de definir, por lo que ha habido una gran cantidad de términos para referirse a ello — superdotado, biendotado, sobredotado, con alta capacidad, alumnos más capaces, sujetos de capacidad superior, precoz, talentoso, genio, prodigio- .

Se considera alumno con talento a aquel que muestra habilidades específicas en áreas muy concretas. Un tipo particular es el talentoso académico o alumno con alto rendimiento; en este se combinan, al menos, recursos elevados de tipo verbal, lógico y de gestión de la memoria (Martínez y Guirardo, 2010). Pero un alumno con talento no tiene por qué ser un AACI.

La Dra. Bertie Kingore (2004), creadora del Inventario de Observación Kingore (especializado en la identificación de niños pequeños y diferenciación en el aula de los AACI), delimitó una serie de categorías que permiten comparar entre estos dos grupos de estudiantes. En la Tabla 1 se recogen algunas de estas características:

Tabla 1. Características diferenciadoras entre niños con alto rendimiento y con altas capacidades. Fuente: Elaboración propia a partir de Kingore (2004)

\begin{tabular}{ll}
\hline \multicolumn{1}{c}{ Con alto rendimiento } & \multicolumn{1}{c}{ Con altas capacidades } \\
\hline Recuerda las respuestas & Plantea preguntas imprevistas \\
Se interesa & Es curioso \\
Está atento & Se concentra mentalmente en forma selectiva \\
Genera ideas avanzadas & Genera ideas complejas y abstractas \\
Rinde en lo más alto del grupo & Está por encima del grupo \\
Aprende con facilidad & Ya sabe \\
Necesita de seis a ocho repeticiones & para \\
dominar un tema & $\begin{array}{l}\text { Necesita de una a tres repeticiones para dominar } \\
\text { Disfruta de la compañía de pares mayores }\end{array}$ \\
Completa las tareas a tiempo & Prefiere la compañía de pares intelectuales \\
Disfruta muchas veces de la escuela & Inicia proyectos y extensiones de las tareas \\
Muy alerta y observador & Disfruta del auto-aprendizaje \\
Satisfecho con su aprendizaje & Anticipa y relaciona las observaciones \\
Obtiene sobresalientes & Es autocrítico \\
\hline & Puede no estar motivado por las notas \\
\hline
\end{tabular}


Ahora bien, ¿cuántos AACI hay? La respuesta a esta pregunta dependerá de la definición que se utilice. En España, el Ministerio de Educación (2013) estima que hay aproximadamente un 5\% del total de la población escolar en enseñanzas de régimen general. Pero en este porcentaje el Ministerio de Educación incluye a los alumnos con alto rendimiento. Otra de las definiciones más seguidas es la que ofrece, por ejemplo, Rodríguez (2009), quien afirma que la capacidad intelectual sigue una distribución estadística normal. Por tanto, será ACCI aquel alumno que se encuentre a dos desviaciones típicas respecto de la media, a su derecha, lo que supone un 2,2\% de la población.

De los 7923293 alumnos matriculados en el sistema educativo español en el curso 2011-2012 en enseñanzas de régimen general —infantil, primaria y Secundaria— sólo a 8605 de estos se les reconoce como AACI, según datos del Ministerio de Educación (2013); es decir, solo se llega a reconocer al 0,108\% de la población, lo cual está muy lejos de ese 2 a $5 \%$ estimado. Además, en los mismos informes realizados en cursos anteriores se obtienen cifras similares.

Esto supone que, en España, aproximadamente entre el 95\% y el 99\% de los escolares con altas capacidades no han sido detectados. Ante esto, nos planteamos la misma cuestión que se realizó la Federación de la Comunidad de Madrid de asociaciones de padres y madres del alumnado (FAPA, 2010): ¿nos encontramos realmente ante un sistema educativo incapaz de detectar a estos alumnos? Lo cual es mucho más grave de lo que aparenta, pues un $70 \%$ de ellos tiene bajo rendimiento escolar, y entre un 35\% y un 50\% fracasa en los estudios (Martín y González, 2000). Parece que a ello han contribuido, entre otras causas, los múltiples estereotipos que giran en torno al alumnado con altas capacidades, no solo por gran parte de la sociedad sino también por buena parte del sector educativo.

Dichos estereotipos pueden definirse como ideas aceptadas comúnmente por un grupo social (Jiménez, 2010), y en este caso constituyen uno de los principales obstáculos para la detección de los AACI. En la Tabla 2 se recogen algunos de los mitos o falsas creencias más comunes.

Tabla 2. Mitos o estereotipos aplicados a los AACI

\begin{tabular}{ll}
\hline \multicolumn{1}{c}{ Mito o estereotipo } & \multicolumn{1}{c}{ La realidad } \\
\hline $\begin{array}{l}\text { Es fácil detectarlos y/o es definitorio obtener un } \\
\text { CI superior a } 130 .\end{array}$ & $\begin{array}{l}\text { La obtención de un CI elevado no puede ser el } \\
\text { único determinante de la alta capacidad. Además, } \\
\text { hace falta una evaluación precisa y colaboración } \\
\text { entre familia y escuela. }\end{array}$ \\
$\begin{array}{ll}\text { Existen más niños que niñas con altas capaci- } \\
\text { dades. }\end{array}$ & $\begin{array}{l}\text { De cada 10 niños identificados, aproximadamen- } \\
\text { te } 3 \text { son niñas, pero los expertos atribuyen esta } \\
\text { diferencia a los patrones sociales y culturales. }\end{array}$ \\
$\begin{array}{ll}\text { Son precoces y desde muy pequeños pueden } \\
\text { mostrar sus características. }\end{array}$ & $\begin{array}{l}\text { No siempre tienen un desarrollo precoz. Algunos } \\
\text { de ellos pueden presentar un desarrollo normal e } \\
\text { incluso tardío. }\end{array}$ \\
$\begin{array}{ll}\text { La mayoría provienen de niveles socioculturales } \\
\text { medios-altos. }\end{array}$ & $\begin{array}{l}\text { Pertenece a cualquier clase social y económica. } \\
\text { Diferente es que reciba más o menos estimulación. } \\
\text { Es un genio. }\end{array}$ \\
\hline
\end{tabular}


Son un grupo homogéneo, con comportamientos Tienen características similares, pero presentan muy similares. tantas diferencias entre sí como el resto de niños.

Son socialmente inadaptados y con problemas Suelen estar adaptados dentro del grupo e inteemocionales. grados socialmente. No obstante, la variabilidad individual es amplia, y se puede encontrar todo tipo de casos.

Tienen problemas para relacionarse con los de Algunos pueden necesitar trabajar estrategias de su edad. relación social, pero no es generalizable. Aunque suelen buscar personas con las que puedan compartir temas de interés y, en ocasiones, las encuentran en mayores.

Son muy solitarios, les gusta estar solos, no Son solitarios cuando las actividades no les intetienen amigos. resan. Los gustos y aficiones suelen ser distintos a las de otros niños de su edad, por lo que no tienen como punto de referencia los juegos y temas de conversación de sus iguales.

Son muy prepotentes.

Esta "prepotencia" viene definida por sus compañeros, e incluso profesores, y se debe, en la mayoría de los casos, a que son niños que utilizan un vocabulario más extenso que los demás, tienen más habilidad para asociar ideas y exponerlas, aprenden más rápido y el resultado de todo esto es que ofrecen una cierta prepotencia solo en apariencia.

Más incidencia de patologías mentales.

Diferentes estudios han comprobado que la incidencia de enfermedades mentales es similar a la del resto de la población.

Triunfa académicamente y en todas las áreas.

Pueden destacar en algún dominio pero no necesariamente en todos, así como algunos pueden llegar a fracasar escolarmente.

Generalmente se aburre en la escuela.

Esto puede suceder si la escuela no responde a sus capacidades, ritmo de aprendizaje, si es repetitiva y rutinaria, y/o no da lugar a la creatividad.

Sus compañeros les critican. Pueden sintonizar perfectamente con sus compañeros y profesores.

Gran motivación para sobresalir en el colegio Esto suele ser lo más identificativo de los talentoy/o alto rendimiento académico. sos académicos. El rendimiento estará en función de la motivación específica que tengan, del estímulo que reciban y del desarrollo procedimental de sus técnicas de trabajo.

Solo rinde en aquello que le gusta (es vago y caprichoso).

Una vez que se adaptan al medio educativo, si este es capaz de satisfacer sus necesidades, no suelen presentar problemas escolares.

Fracaso escolar. La desmotivación, la falta de hábitos de estudio o la igualación de competencias intelectuales a sus compañeros pueden conducirlo al fracaso escolar; pero si tienen un adecuado entorno educativo, no tiene por qué darse el fracaso escolar. 


\begin{abstract}
Avanza por sí mismo y puede lograr el éxito aca- Aprenden rápido y con facilidad, pero necesitan démico sin necesidad de ayuda. que se les oriente, apoye y estimule.

Aprenden solos.

No aprenden por "arte de magia", siempre tiene que haber alguien que les enseñe, que les explique, que les ayude a procesar una información, que les prepare para estudiar. Uno de los problemas principales de la adolescencia de estos niños es la falta de hábitos de estudio y trabajo y, para ello, es muy importante la figura del adulto.

Son tan inteligentes que los profesores no esta- El profesor no debería centrar su papel en la sumos preparados. perioridad de conocimientos, sino en una mayor madurez y un mejor conocimiento de procedimientos de aprendizaje.
\end{abstract}

Fuente: Adaptado de Martín y González (2000), Fernández y Sánchez (2010), Martínez y Guirardo (2010) y Albes (2013).

Vencer este tipo de mitos o estereotipos resulta esencial para efectuar una adecuada identificación de un alumno con ACI, a fin de ofrecerle una respuesta educativa adaptada a sus necesidades. Es decir, el fin de este diagnóstico debe ser siempre mejorar su educación, ya que "observar su manera de ser y de aprender es parte de nuestra tarea como docentes" (Martínez y Guirardo, 2010: 33). La mera etiqueta, si no se encuentra aparejada a un cambio en las prácticas escolares que beneficie a la formación del estudiante, no sirve de nada.

Además, la mayoría de expertos considera que la detección de estos alumnos debe realizarse cuanto antes, entendiendo que es necesario identificar al AACI para poder conocer sus características y funcionamiento intelectual personales y hacer propuestas acordes a sus necesidades (Albes, 2013).

En esta línea, Alonso y Benito (1996) aseguran que la importancia de la identificación temprana es primordial, aludiendo a las conclusiones que Bárbara Clark obtuvo tras un estudio sobre el daño neuronal a causa de la no utilización del potencial humano:

Figura 1. Consecuencias de la no utilización del potencial humano

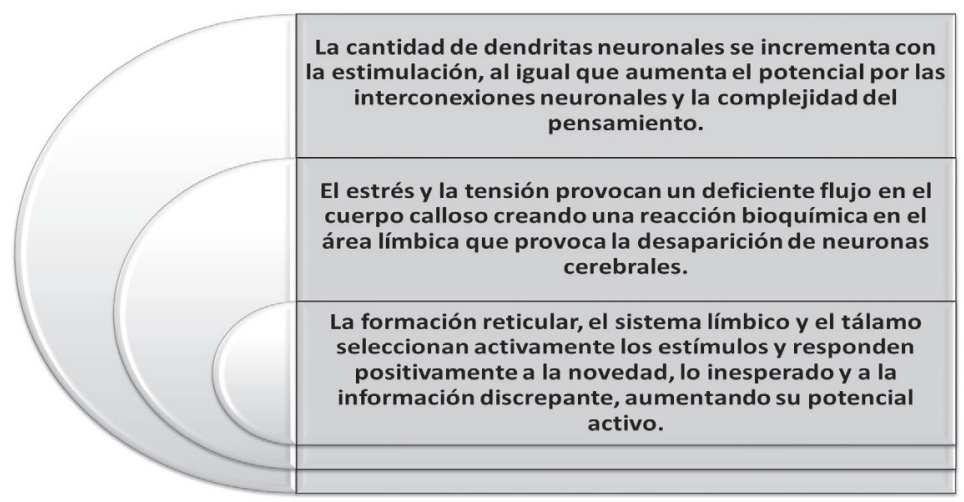

Fuente: Elaboración propia. 
En base a estos hechos, dichos autores subrayan que la ausencia de una estimulación adecuada ocasiona no solo que los alumnos no desarrollen plenamente todo su potencial, sino que la tensión y el estrés que ello les provoca ocasione una merma en este.

Otra gran problemática en relación a la educación y formación integral de estos alumnos consiste en que actualmente los centros educativos no disponen de una oferta educativa lo suficientemente diversificada, estando pensada esta para los alumnos medios (Martín y González, 2000).

La respuesta educativa de cualquier escolar, no sólo del AACI, debe ser un conjunto de acciones relacionadas que concreten las opciones de enseñanza que se llevan a cabo en el contexto escolar, con el objetivo de potenciar todas las capacidades de los alumnos. A lo largo de las últimas décadas se han producido muchos cambios respecto a la educación del alumnado con necesidades educativas de apoyo específico. La escuela inclusiva parte de la idea de que cada alumno es distinto y, por tanto, debe darse también una respuesta educativa diferenciada (Martínez y Guirardo, 2010).

\section{Figura 2. Medidas ordinarias, extraordinarias y excepcionales}

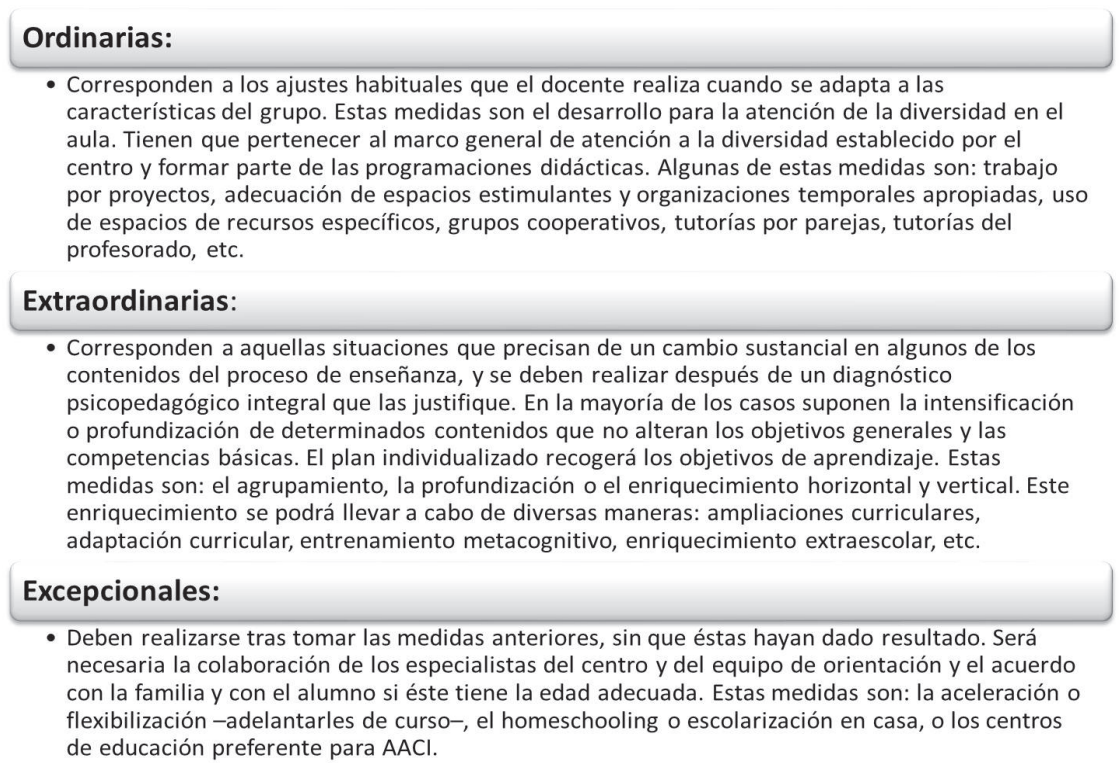

Fuente: Elaboración propia.

Evidentemente, las oportunas medidas educativas sólo se podrán dar al AACI que ya ha sido identificado, y ya hemos visto que su número es lamentablemente reducido. En estos casos, se debe actuar desde el primer momento en que se tenga alguna sospecha de la existencia de altas capacidades. Sin embargo, la heterogeneidad de este alumnado es tan amplia que serán muy diferentes las medidas educativas que se podrán adoptar en cada caso (Apraiz de Elorza, 1994). Por tanto, no existen medidas eficaces por sí mismas, 
sino aplicaciones de estas para unos determinados alumnos y en los contextos concretos. Así, una misma intervención puede ser eficaz para un alumno determinado pero no para otros. De ello se deduce que los resultados dependerán también de la familia e incluso del grupo-clase (Martínez y Guirardo, 2010). A continuación se diferencian las medidas que se pueden tomar, dividiéndolas en ordinarias, extraordinarias y excepcionales (Apraiz de Elorza, 1994; Expósito, 2012; Fernández y Sánchez, 2010).

Además de estas medidas, entre las respuestas educativas que últimamente se están desarrollando para acomodar el nivel de enseñanza y los modos de aprender del AACI, se está apostando por el uso de las tecnologías de la información y la comunicación y los dispositivos móviles en el aula (Hernández y Gutiérrez, 2014; Tourón y Tourón, 2011).

Sin embargo, toda iniciativa o medida que se lleve a cabo, así como la implementación de una correcta y temprana detección, están sujetas al fracaso si el profesorado no cuenta con la preparación y los conocimientos necesarios sobre el AACI. De hecho, son numerosos los estudios que avalan la enorme importancia que adquiere la formación del profesorado para identificar y atender adecuadamente a estos alumnos (Hernández y Gutiérrez, 2014), delimitándose incluso determinadas competencias docentes específicas para ello (Conejeros, Gómez y Donoso, 2013).

\section{MÉTODO}

La presente investigación comenzó con una exhaustiva revisión bibliográfica en el tema que nos ocupa, examinando cuáles son las tendencias actuales en cuanto al concepto y a los conocimientos que se tienen sobre las altas capacidades.

Tras ello, se hizo un estudio empírico descriptivo y multivariante con el objetivo de contrastar los conocimientos actuales con la percepción que tiene el profesorado de educación secundaria respecto a los AACI. Este estudio estuvo dirigido en un principio a todos los docentes de secundaria, pero sorprendentemente una cifra bastante considerable de los participantes, el 27,63\%, reconocieron haber tenido formación específica respecto a este alumnado en cuestión. Ante este hecho, y teniendo en cuenta que una de las problemáticas que se encuentra al sistema educativo es la escasa preparación del profesorado en necesidades educativas, se decidió separar el estudio en dos grupos: aquellos docentes que no tienen esta formación específica, y los que sí la tienen, a fin de comparar el enfoque de sus respuestas.

\subsection{MUESTRA}

El tamaño de la muestra inicial fue de 691 participantes. Tras la depuración de la matriz de datos, descartando los valores perdidos, se procedió a realizar un análisis exploratorio EDA (Exploratory Data Analysis) que permitió detectar los outliers y obtener una muestra final compuesta por 637 sujetos procedentes de todo el territorio español, de los cuales 176 tenían formación específica en las necesidades del AACI.

\subsection{INSTRUMENTO}

La recogida de información se realizó mediante el método de la encuesta online. El instrumento elaborado ad hoc para ello fue un cuestionario construido en Google Drive 
(drive.google.com). El principal objetivo del diseño del cuestionario fue examinar si los conocimientos actuales se corresponden con la realidad de la práctica educativa de los docentes. Para ello, se comprobará si se han superado ya los tradicionales estereotipos que se aplican a este colectivo, así como si se conocen las actuales medidas educativas ordinarias, extraordinarias y excepcionales — que se les puede aplicar.

Se trata de un cuestionario compuesto por 32 ítems, en un solo bloque. Los cinco primeros ítems se corresponden con información exploratoria: edad, género, comunidad autónoma, profesor de educación secundaria y tipo de centro escolar; hay también 14 ítems de respuesta dicotómica — Sí/No—, 12 ítems de elección múltiple y un ítem de respuesta abierta.

Para asegurar la validez del cuestionario se emplearon las siguientes técnicas:

Figura 3. Validez del cuestionario

\section{Validez de contenido:}

- Se solicitó a ocho jueces expertos que hiciesen una valoración cualitativa y cuantitativa (escala de 1 a 10) de diferentes aspectos sobre la información inicial, la escala de medida, y los ítems del cuestionario, concretamente de: a) el grado de pertenencia al objeto de estudio, registrando qué ítems debían formar parte del mismo; y b) del grado de precisión y corrección, registrando la precisión de la definición y redacción de cada uno de los ítems.

\section{Validez de comprensión:}

- Se analizó mediante autoinforme el grado en el que los docentes entendían los diferentes ítems del cuestionario, registrando de forma cualitativa todas aquellas dudas o sugerencias que los docentes señalaron acerca de su cumplimentación.

Fuente: Elaboración propia.

\subsection{PROCEDIMIENTO}

El muestreo de este estudio se puede considerar no probabilístico por cuotas, ya que la difusión del cuestionario se realizó mediante envío directo por correo electrónico del enlace de acceso al mismo a las secretarías de los centros de educación secundaria del territorio español. La búsqueda de estas direcciones de correo electrónico se realizó a través de la web de la Consejería de Educación.

En total se remitieron unos 290 correos, cada uno de los cuales fue enviado a 18 centros diferentes, alcanzándose un total aproximado de 5000 centros de educación secundaria.

Tras completar este envío, y a fin de incrementar el alcance de la encuesta, el enlace de acceso al cuestionario fue publicado en grupos de docentes presentes en diversas 
redes sociales, como Facebook y LinkedIn, dirigiéndose al grupo de docentes objeto de estudio (docentes de secundaria). Este paso imprime al muestreo un carácter aleatorio por conglomerados, que añade mayor representatividad a la muestra.

El cuestionario estuvo disponible aproximadamente durante un mes. Al cierre de este, se realizó un análisis estadístico descriptivo y multivariante de los resultados obtenidos, utilizando para ello el programa Statgraphics Centurion.

\section{RESULTADOS}

\subsection{CARACTERÍSTICAS DE LA MUESTRA}

Ha habido una mayor participación de mujeres que de hombres: un 63,11\% frente a un $36,89 \%$. Asimismo, el rango de edad mayoritaria se sitúa entre los 40 y 49 años, con un $43,33 \%$, seguido de los que tienen entre 50 y 60 años, con un 30,91\%. Además, en cuanto a la relación entre género y edad, el grupo constituido por mujeres de entre 40 y 49 años es el que ha tenido una mayor participación. Del total de la muestra, el 86,97\% eran docentes de centros públicos y el 13,03\% de centros privados o concertados.

\subsection{EXPERIENCIA Y FORMACIÓN DE LOS PARTICIPANTES}

El 68,45\% de los encuestados reconocieron haber tenido algún alumno con altas capacidades intelectuales, lo cual parece ser un hecho muy llamativo pues, tal y como se explicó en la introducción, entre un $95 \%$ y un $99 \%$ del AACI pasa inadvertido por las aulas. Ante esto, hemos de argumentar que, al correo de referencia, recibimos algunas respuestas del tipo: "Lo que pasa es que nunca he tenido a uno de estos alumnos, por lo que no sé si servirá de algo responderlo". Así pues, estimamos que la baja participación de profesores que no han tenido alumnos con altas capacidades intelectuales se debe a motivos similares. Además, de estos alumnos, el 64,91\% fue detectado en Secundaria.

Respecto a la formación del profesorado, el 27,63\% reconoce haber tenido formación específica en alumnos con altas capacidades intelectuales.

A continuación, vamos a examinar algunos datos referentes a los estereotipos de índole académica con los que se identifica a estos alumnos.

\subsection{PERCEPCIONES RELACIONADAS CON EL APRENDIZAJE}

Ante la pregunta de si se considera la precocidad como un rasgo distintivo, el resultado es prácticamente el mismo, tanto si se considera que el profesor tiene formación específica $(51,70 \%)$ como si no $(48,30 \%)$.

En cuanto a si se considera que los AACI son alumnos que están siempre comprometidos con la tarea, los resultados son similares, tanto como en el conjunto del profesorado sin formación como en el que sí tiene formación, con un 93,72\% y un 93,75\%.

Si el ítem analizado es si se considera que el niño con altas capacidades intelectuales ha de destacar en todo, el resultado es similar al ítem previo (el AACI está siempre comprometido con la tarea), con un $94,35 \%$ correspondiente al profesorado sin formación que opinan que no, y un 94,32\% correspondiente al profesorado con formación. 
$\mathrm{Al}$ tener en cuenta el rendimiento de los AACI, el 27,32\% opina que solo rinden en aquellas asignaturas que les gustan, el 5,65\% opina que son estudiantes aplicados y comprometidos con su aprendizaje, el 4,55\% piensa que son estudiantes problemáticos y que se aburren en clase. Entre estas tres diferentes opiniones, que recordemos que se corresponden a estereotipos, tenemos un 37,52\% del total de los encuestados. El 62,48\% no está de acuerdo con ninguna de las anteriores opiniones. Al analizarlos teniendo en cuenta que sí posean formación, el 30,10\% está de acuerdo con algunas de estas opiniones estereotipadas.

Por otra parte, el 70,50\% de los profesores que no tienen formación piensa que las capacidades de este alumnado sí son innatas; y esto mismo lo piensan un $65,34 \%$ de los profesores con formación.

Otro estereotipo es considerar que el coeficiente de inteligencia, medido con escalas que no siempre son oportunas, es el factor determinante para saber si un alumno es o no un AACI. El 21,19\% de los docentes sin formación considera que si en esta medida el niño presenta un CI superior a 130, entonces ya se le puede considerar como AACI. Un $78,65 \%$ considera que, además, son necesarios otros factores y un $0,16 \%$ opina que este factor nunca puede ser determinante para decidir si un niño tiene o no altas capacidades. Considerando a aquellos profesores con formación, el 19,32\% sostiene que el que un alumno muestre un coeficiente superior a 130 en alguna determinada escala ya es suficiente para considerarlo con altas capacidades intelectuales, pero el $80,11 \%$ opina que esto no ha de ser el factor determinante.

Además, el 49,92\% de los profesores sin formación no sabe diferenciar entre un AACI y un alumno de alto rendimiento; al igual que tampoco son capaces de diferenciar ambos tipos de alumnos el 12,50\%, incluso teniendo formación.

Tras estos datos, se analizan los ítems referentes al factor social en este alumnado.

\subsection{PERCEPCIONES ACERCA DE LA SOCIALIZACIÓN}

La mitad del profesorado piensa que los compañeros del AACI suelen rehuir de él, exactamente el 50,08\% del profesorado sin formación y el 45,45\% de los profesores que sí han tenido formación.

Respecto al ítem de si se relaciona preferentemente con personas de mayor edad que él, el 37,68\% del profesorado sin formación opina que así es. Este porcentaje también es parecido al considerar solo a aquellos que han recibido formación específica: el 35,80\%.

En cuanto al tipo de relación que mantiene con sus compañeros de aula, el 59,65\% del profesorado han respondido que el tipo de relación que un AACI mantiene con sus compañeros es similar a cualquier tipo de relación de compañeros. Sin embargo, el 32,03\% afirma que no se relaciona bien con sus compañeros y, en contraposición, el 8,23\% dice que sí tiene buena relación. Al analizar este ítem teniendo en cuenta la formación específica del profesorado, los resultados son similares: $64,20 \%, 26,70 \%$ y $9,09 \%$ respectivamente.

Por otra parte, el $87,76 \%$ del profesorado opina que no es necesario que los AACI se relacionen entre ellos. Esta cifra es similar, con un $85,80 \%$ si se considera al grupo que posee formación específica.

Al analizar si se da prioridad al estado emocional o a la enseñanza-aprendizaje, el $64,68 \%$ afirma que su estado emocional debe ser la prioridad, al igual que el 71,02\% del profesorado con formación. 
A continuación se analizarán los datos obtenidos respecto a las necesidades y respuestas educativas que requiere este alumnado.

\subsection{PERCEPCIONES ACERCA DE LA RESPUESTA EDUCATIVA}

Prácticamente, el profesorado en general, un 92,31\%, reconoce que el AACI, además de presentar las necesidades educativas comunes de cualquier otro niño, también presenta necesidades educativas específicas por sus determinadas características. Sin embargo, a pesar de que se sabe que posee necesidades educativas, solo el 38,49\% del profesorado sin formación sí sabe cuáles son; esta cifra se eleva considerablemente al 77,71\% al considerar la formación del profesorado como referente. De todas formas, el 22,29\% de los docentes que sí tienen formación reconocen que no saben cuáles son las necesidades educativas de estos estudiantes.

Ahora bien, a pesar de que el 92,31\% reconocía que el AACI presenta necesidades educativas específicas por sus determinadas características, si la pregunta es si necesita una educación personalizada aunque no presente dificultades de aprendizaje, entonces solo el $66,72 \%$ sigue sosteniendo que son necesarias medidas educativas específicas. Incluso el 25,57\% del profesorado con formación específica opina que si el niño con altas capacidades intelectuales no presenta dificultades de aprendizaje entonces no es necesario tomar ninguna medida en particular.

Siguiendo con el análisis sobre las opiniones sobre las necesidades educativas, el $53,53 \%$ opina que este alumno puede necesitar ayuda en determinadas materias y el 43,49\% afirma que todos necesitan algún tipo de ayuda. El 2,98\% piensa que no necesitan ninguna ayuda, ya que son muy inteligentes. Si se considera solo a aquellos que han recibido formación, entonces los resultados son que el 60,80\% opina que puede necesitar ayuda en determinadas materias y el 36,93\% afirma que todos los AACI necesitan algún tipo de ayuda. Pero sigue habiendo un 2,27\% que piensa que no necesitan ninguna ayuda, ya que son muy inteligentes.

En el ítem 26 del cuestionario se ofrecieron varias posibilidades para atender sus necesidades educativas. Al considerar ambos grupos por separado, aquellos sin y con formación, el resultado vuelve a ser similar, con más del $90 \%$ afirma que la respuesta educativa que se le debe dar depende de sus propias necesidades y en función de la asignatura.

En la Tabla 3 recogemos de forma resumida los resultados más relevantes anteriormente expuestos: 
Tabla 3. Resumen de los resultados más relevantes

\begin{tabular}{lcc}
\hline & $\begin{array}{c}\text { Profesores sin } \\
\text { formación }\end{array}$ & $\begin{array}{c}\text { Profesores con } \\
\text { formación }\end{array}$ \\
\hline La precocidad es un rasgo distintivo & $51,70 \%$ & $48,30 \%$ \\
Están siempre comprometidos con la tarea & $93,72 \%$ & $93,75 \%$ \\
Destacan en todo & $94,35 \%$ & $94,32 \%$ \\
Sus capacidades son innatas & $70,50 \%$ & $65,34 \%$ \\
Los compañeros suelen rehuir de ellos & $50,08 \%$ & $45,45 \%$ \\
Se relaciona preferentemente con personas mayores & $37,65 \%$ & $35,80 \%$ \\
$\begin{array}{l}\text { La relación con sus compañeros de aula es similar a la de } \\
\text { cualquier otro alumno }\end{array}$ & $59,65 \%$ & $64,20 \%$ \\
$\begin{array}{l}\text { No es necesario que los AACI se relacionen entre ellos } \\
\text { Su estado emocional debe ser la prioridad }\end{array}$ & $87,76 \%$ & $85,80 \%$ \\
$\begin{array}{l}\text { Presenta necesidades educativas específicas, pero el } \\
\text { docente no sabe cuáles son }\end{array}$ & $64,68 \%$ & $71,02 \%$ \\
$\begin{array}{l}\text { Presenta necesidades educativas específicas, aunque no } \\
\text { tenga dificultades de aprendizaje }\end{array}$ & $61,51 \%$ & $22,29 \%$ \\
\hline
\end{tabular}

Fuente: Elaboración propia.

A continuación, mostramos en la Tabla 4 los resultados respecto a las posibles medidas en la adaptación curricular individualizada:

Tabla 4. Opiniones sobre la adaptación curricular individualizada

\begin{tabular}{lcc}
\hline & $\begin{array}{c}\text { Profesores sin } \\
\text { formación }\end{array}$ & $\begin{array}{c}\text { Profesores con } \\
\text { formación }\end{array}$ \\
\hline $\begin{array}{l}\text { Debe adaptarse el currículo con conocimientos de otros } \\
\text { cursos }\end{array}$ & $48,98 \%$ & $45,45 \%$ \\
$\begin{array}{l}\text { Debe adaptarse el currículo pero no incluir más } \\
\text { conocimientos }\end{array}$ & $20,88 \%$ & $16,48 \%$ \\
$\begin{array}{l}\text { Si el alumno tiene un buen progreso, la adaptación no es } \\
\text { necesaria }\end{array}$ & $30,14 \%$ & $38,07 \%$ \\
\hline
\end{tabular}


Una de las medidas más polémicas es la aceleración. En la Tabla 5 se recogen las opiniones vertidas en este estudio:

Tabla 5. Opiniones sobre la aceleración

\begin{tabular}{lcc}
\hline & $\begin{array}{c}\text { Profesores sin } \\
\text { formación }\end{array}$ & $\begin{array}{c}\text { Profesores con } \\
\text { formación }\end{array}$ \\
\hline $\begin{array}{l}\text { Aceptable, así el ACCI podrá tener antes estudios } \\
\text { superiores }\end{array}$ & $8,16 \%$ & $6,25 \%$ \\
$\begin{array}{l}\text { Aceptable, pero aún así el AACI necesita adaptaciones } \\
\text { curriculares }\end{array}$ & $36,73 \%$ & $36,36 \%$ \\
$\begin{array}{l}\text { Bueno, así se pedirá al AACI el mismo nivel que a sus } \\
\text { compañeros }\end{array}$ & $4,71 \%$ & $4,55 \%$ \\
$\begin{array}{l}\text { Es mala medida, no conviene sacar al AACI del grupo de } \\
\text { su misma edad }\end{array}$ & $25,59 \%$ & $23,30 \%$ \\
$\begin{array}{l}\text { Sin importancia, no afecta a la calidad educativa que } \\
\text { recibe el AACI }\end{array}$ & $7,85 \%$ & $4,55 \%$ \\
\begin{tabular}{l} 
Otro \\
\hline
\end{tabular} & $16,95 \%$ & $25 \%$ \\
\hline
\end{tabular}

Ya que la aceleración o flexibilización es una de las medidas a adoptar más polémica, en este ítem se dio la opción de "otro", de modo que los encuestados pudieran expresar y comentar cualquier opinión al respecto. En el Anexo se ha incluido un resumen de dichas opiniones, analizadas y categorizadas en torno a cinco variables:

- Opiniones tales que consideran la educación del AACI de forma personalizada.

- Opiniones tales que consideran que no es una medida adecuada.

- Opiniones tales que consideran que es una medida aceptable.

- Opiniones tales que consideran que es una buena medida.

- Opiniones tales que consideran que es mejor que el AACI continúe con su grupo de referencia.

Finalmente, en el ítem 27 se ha analizado cuál sería el tipo de escuela en donde estos alumnos pueden recibir una mejor educación. Los resultados se muestran en la Tabla 6: 
Tabla 6. Opiniones sobre cuál es el mejor centro educativo

\begin{tabular}{lcc}
\hline & $\begin{array}{c}\text { Profesores sin } \\
\text { formación }\end{array}$ & $\begin{array}{c}\text { Profesores con } \\
\text { formación }\end{array}$ \\
\hline Un centro de educación especial & $0,47 \%$ & $0 \%$ \\
Un centro ordinario & $20,25 \%$ & $27,84 \%$ \\
Un centro ordinario, pero con adaptaciones curriculares & $58,24 \%$ & $60,80 \%$ \\
Un centro preferente para AACI & $21,04 \%$ & $11,36 \%$ \\
\hline
\end{tabular}

Fuente: Elaboración propia.

Al término del cuestionario, se dio la oportunidad a los participantes de remitir alguna consideración que estimasen importante respecto a los AACI, y de todas las respuestas recibidas en este ítem de carácter abierto, se exponen algunas en el Anexo presentado al final del presente artículo. Dichas consideraciones fueron analizadas y categorizadas en torno a las siguientes variables:

- Comentarios que implican la labor del profesor, su formación y al sistema educativo en general.

- Comentarios relacionados con la detección del AACI.

- Comentarios respecto a las respuestas educativas extraordinarias y excepcionales.

- Comentarios sobre las emociones y socialización en los AACI.

- Comentarios respecto al AACI como individuo, y no como grupo.

- Comentarios que inciden en otros agentes.

\section{CONCLUSIONES}

Este trabajo ha permitido realizar una aproximación a la opinión y el conocimiento que tienen actualmente los profesores de educación secundaria en España respecto al AACI, aspecto de vital importancia puesto que en las aulas de secundaria solo se consigue identificar en torno al 0,1\% de estos estudiantes, cuando se estima que existe entre un $2 \%$ y un 5\%. Además, un alto número de ellos fracasa en los estudios e incluso los abandonan, motivo por el cual resulta interesante saber qué preparación tienen los profesores sobre este alumnado y su educación.

Como se ha podido apreciar en el análisis de los resultados, aún prevalecen muchos de los mitos que se adjudican a estos jóvenes, dotándolos de un perfil estereotipado que nada tiene que ver con la realidad. Precisamente, Martín y González (2000) sostienen que es este el principal problema a la hora de diagnosticar con altas capacidades intelectuales a aquellos estudiantes que realmente las tengan.

Otro aspecto importante es detectar cuanto antes a estos alumnos para poder ofrecerles lo más prontamente posible las ayudas que necesitan. Sin embargo, a partir de las respuestas obtenidas en el presente estudio por parte de aquellos encuestados que han tenido a uno 
de estos jóvenes en sus clases, se puede observar que 7 de cada 10 alumnos con ACI no llegaron a ser detectados hasta la etapa de secundaria. Además, aunque la mayoría de profesores haya reconocido en sus respuestas que dichos estudiantes tienen necesidades educativas específicas debido a sus altas capacidades, muy pocos saben cuáles son dichas necesidades y qué implican.

Por otra parte, un alto porcentaje de los participantes ha afirmado tener formación específica para atender al AACI. Pese a ello, al analizar sus respuestas sorprende encontrar que sus conocimientos resultan similares a los que muestra el profesorado sin dicha formación. Esto coincide con los resultados obtenidos por parte de otros investigadores, como por ejemplo Peña et al. (2003), quienes encontraron en su estudio que los profesores tienen dificultades para valorar algunas de las características que presentan los estudiantes con altas capacidades según la literatura científica. En razón a ello, se observa que en este sentido algo está fallando, por lo que sería conveniente averiguar si la formación que se está impartiendo sobre este tipo de alumnado resulta realmente apropiada.

Por último, y como ya se ha puesto de relieve a lo largo del presente artículo, la formación inicial y permanente del profesorado resulta esencial tanto para romper con los estereotipos detectados, como para hacer un correcto diagnóstico y atender adecuadamente las necesidades educativas de los AACI. Solamente se puede avanzar en su detección y mejorar su educación si el sistema apuesta por dotar a sus centros y docentes de los recursos necesarios para ello. Por esta razón se desea concluir el presente artículo destacando esta urgencia y haciendo una llamada a las autoridades educativas para que pongan los medios a su alcance para solucionar esta situación.

\subsection{LIMITACIONES DEL ESTUDIO}

Una de las principales limitaciones que presenta este trabajo es el instrumento de recogida de información utilizado, elaborado ad hoc, cuya fiabilidad y validez podrían mejorarse ampliando las pruebas psicométricas y estadísticas pertinentes. Asimismo, una debilidad de este estudio consiste en su muestra, que pese a ser bastante amplia, no resulta significativa respecto al total de individuos que configura la población de referencia. En consecuencia, los resultados obtenidos no pueden considerarse completamente extrapolables y deben interpretarse con cautela, evitando posibles errores de inferencia.

\subsection{PROSPECTIVA}

Este trabajo pone de relieve la necesidad de seguir investigando acerca de las percepciones que el profesorado de secundaria presenta acerca de los AACI. Por ello, en primer lugar se propone efectuar un estudio más exhaustivo, con un instrumento de recogida de información más complejo, a partir del cual se pueda obtener una muestra mayor, más significativa y representativa.

En vista de los resultados, sería conveniente indagar en la formación ofrecida al profesorado en materia de atención al AACI en particular, y en materias de atención a la diversidad en general, tanto en la formación universitaria como en los cursos de actualización de competencias del profesorado o cualquier otro tipo de formación recibida.

Otra línea interesante consistiría en realizar una comparativa con la percepción que tiene el profesorado de otros países acerca de este alumnado, la atención educativa que 
se le proporciona, y las medidas, estrategias y recursos que se le ofrecen, a fin de tomar referencias que sirvan de ejemplo de buenas prácticas y hagan posible mejorar la situación de los AACI en nuestro país.

\section{REFERENCIAS BIBLIOGRÁFICAS}

Albes, C. (2013). Orientaciones educativas: alumnado con altas capacidades. Vitoria: Servicio Central de Publicaciones del Gobierno Vasco.

Alonso, J. A., \& Benito, Y. (1996). Superdotados: Adaptación escolar y social en Secundaria. Madrid: Narcea.

Apraiz de Elorza, J. (1994). La educación del alumno con altas capacidades. Vitoria: Servicio Central de Publicaciones del Gobierno Vasco.

Conejeros, M. L., Gómez, M. P., \& Donoso, E. (2013). Perfil docente para alumnos/as con altas capacidades. MAGIS, Revista Internacional de Investigación en Educación, 5(11), 393-411.

Expósito, M. M. (2012). Medidas de intervención específicas para alumnos con altas capacidades en la Comunidad de Madrid: Respuestas educativas y programa de enriquecimiento. En J. C. Torrego (Coord.), Alumnos con altas capacidades y aprendizaje cooperativo. Un modelo de respuesta educativa (pp. 53-88). Madrid: Fundación Pryconsa y Fundación SM.

FAPA. (2010). Informe sobre la situación de la atención educativa del alumnado con altas capacidades. Madrid: Autor. Recuperado desde: http://www.fapaginerdelosrios.org/index.php $? \mathrm{~m}=$ Documentos\&op $2=$ descargar \&did $=56$

Fernández, M. T., \& Sánchez, M. T. (2010). Cómo detectar y evaluar a los alumnos con altas capacidades. Guía para profesores y orientadores. Sevilla: Psicoeduca.

Hernández, D., \& Gutiérrez, M. (2014). El estudio de la alta capacidad intelectual en España: análisis de la situación actual. Revista de Educación, 364, 251-272. doi:0.4438/1988-592XRE-2014-364-261

Jiménez, C. (2010). Diagnóstico y educación de los más capaces. Madrid: Pearson Educación.

Kingore, B. (2004). Differentiation: Simplified, Realistic, and Effective. Austin (Texas): Professional Associates Publishing.

Martín, J., \& González, M. (2000). Alumnos precoces, superdotados y de altas capacidades. España: Secretaría General Técnica. Centro de publicaciones.

Martínez, M., \& Guirardo, A. (2010). Alumnado con altas capacidades. Barcelona: Graó.

Miguel, A., y Moya, A. (2012). Conceptos generales del alumno con altas capacidades. En J. C. Torrego (Coord.), Alumnos con altas capacidades y aprendizaje cooperativo. Un modelo de respuesta educativa (pp. 13-34). Madrid: Fundación Pryconsa y Fundación SM.

Ministerio de Educación. (2013). Informe sobre el estado y situación del sistema educativo. Curso 2011/2012. España: Secretaria General Técnica.

Peña, A. M., Martínez, R. A., Velázquez, A. E., Barriales, M. R., \& López, L. (2003). Estudio de las características que percibe el profesorado en alumnos con alta capacidad intelectual. Revista de Investigación Educativa, 21, 271-289.

Rodríguez, L. (2009). Alta capacidad intelectual. Recuperado desde: http://formacioniesmelide. weebly.com/uploads/1/2/4/9/12493581/09as_altas_capacidadesluis_rodriguez_cao.pdf

Tourón, J., \& Tourón, M. (2011). The Center for Talented Youth Identification Model: A review of the literature. Talent Development \& Excellence, 3, 187-202. 


\begin{abstract}
ANEXO
Opiniones de los participantes respecto al ítem 26:

A continuación se muestra un resumen de las opiniones vertidas por los encuestados acerca de la aceleración como medida para atender las necesidades educativas de los AACI, categorizadas en torno a cinco variables:

a. Opiniones tales que consideran la educación del AACI de forma personalizada.

b. Opiniones tales que consideran que no es una medida adecuada.

c. Opiniones tales que consideran que es una medida aceptable.

d. Opiniones tales que consideran que es una buena medida.

e. Opiniones tales que consideran que es mejor que el AACI continúe con su grupo de referencia.

- Opiniones tales que consideran la educación del AACI de forma personalizada:

- "Cada alumno con altas capacidades posee unas características diferentes. También hay mucha diversidad en este tipo de alumnado".

- "Cada caso debería tratarse de forma individual y realizar un seguimiento de la medida adoptada".

- "Depende de su madurez afectiva, del pronóstico de integración social y, sobre todo, de las necesidades específicas que tenga".

- "Se trata de que esté a gusto con sus compañeros".

- "Depende del caso concreto (características intelectuales, sociales y emocionales), y en todo caso, debe acompañarse de otras medidas".

- "Es una medida que hay que estudiar para cada caso de manera individualizada para ver si emocionalmente se beneficia".

- "Es una medida que se puede adoptar siempre y cuando se adapte a las características del alumno y se piense que puede responder a las necesidades que presenta ese alumno tras la evaluación psicopedagógica pertinente".

- "TODOS presentan características personales. Sólo hay que proporcionarles aquello que precisan, como con cualquier alumno. ¿Qué precisan? Eso dependerá de cada caso".
\end{abstract}

- Opiniones que consideran que no es una medida adecuada:

- "Creo que es mucha la burocracia que hay que completar para conseguir adelantarles un curso. La realidad es que no suele haber apoyos para los AACI y, sin embargo, sí la hay para los alumnos a los que les cuesta muchísimo seguir el ritmo habitual de una clase".

- $\quad$ "Debe ser una medida excepcional ya que normalmente quedan etiquetados y eso es negativo. El sistema educativo debería ser más flexible para todos".

- "Es la medida más barata, pero no permitirá que los niños desarrollen su potencial".

- "Sería mejor que estuvieran en un centro totalmente bilingüe o trilingüe y con adaptaciones curriculares".

- "Lo que se debería hacer es dotar de recursos a los centros para tratar a estos alumnos sin que los compañeros los vean como los raros".

- "Profesores motivados y grupos reducidos integrando AACI con alumnos de alto rendimiento". 
- "Sería más conveniente que recibiera su formación en un centro específico que se adapte completamente a sus necesidades".

- Opiniones tales que consideran que es una medida aceptable:

- "Es una medida aceptable en algunos casos. Hay que tener en cuenta la madurez personal del alumno".

- "Es una medida aceptable pero aún así el AACI necesita adaptaciones y es preferible que se realice en los cursos más bajos".

- $\quad$ "Es una medida aceptable siempre y cuando se posibiliten otras necesidades del alumno".

- Es una medida aceptable sólo si se aburre tanto en clase que acaba comportándose mal; es mejor que se trate con niños de su edad y aprenda habilidades sociales a su mismo ritmo".

- "Es una medida aceptable ya que se le exige el mismo nivel académico que al resto de los compañeros pero la diferencia de edades podría generar problemas de adaptación con sus compañeros".

- "Es una medida correcta si se hace al inicio de una etapa y a las AACI se le une la madurez del alumno".

- Opiniones tales que consideran que es una buena medida:

- "Es una buena medida por evitar que se aburra con la facilidad de las clases, pero sólo si él lo prefiere, no si le va a costar ser el intruso o el raro o si tiene que dejar su grupo y ello le duele".

- "Es una buena medida porque así el AACI y su familia, puede elegir adelantar un curso si ellos lo consideran conveniente".

- "Es una buena medida si se prepara al alumno para dar el paso, se da una explicación a los compañeros del curso que deja y a los del que lo reciben y se supervisa su adaptación en el nuevo curso hasta su completa integración".

- "Es una buena medida siempre que no afecte a su proceso de socialización, y siempre después de haber planteado las adaptaciones necesarias con su grupo de referencia".

- "Puede ser una buena medida si el alumno domina los objetivos establecidos para el curso que no cursa y si la adaptación del alumno al nuevo grupo es buena".

- Opiniones tales que consideran que es mejor que el AACI continúe con su grupo de referencia:

- "Considero que estos alumnos deben ampliar en horizontal, dentro de su nivel y con sus compañeros pero habrá que trabajar su motivación para evitar una actitud de aburrimiento".

- "El AACI debe permanecer en el entorno de los compañeros de su edad recibiendo paralelamente una adaptación curricular de ampliación".

- "No lo considero recomendable a nivel social y emocional. En el grupo que le corresponde por edad, se debe garantizar la adecuación curricular y metodológica que estimule y fomente su desarrollo integral, de modo que satisfaga las necesidades y las motivaciones del AACI".

- "No sé si a nivel emocional es bueno o conveniente que se le separe del grupo de su misma edad, aunque tenga un nivel para seguir el ritmo del nuevo grupo". 
- "Se le puede dejar con los compañeros de su misma edad y hacerle trabajar más cosas pidiéndole ayuda para sus compañeros".

Ítem final, abierto a cualquier consideración de los encuestados:

A continuación se recogen algunas de las consideraciones que los participantes hicieron llegar al término del cuestionario. Dichas opiniones han sido analizadas y categorizadas en torno a seis variables:

a. Comentarios que implican la labor del profesor, su formación, y al sistema educativo en general:.

b. Comentarios respecto a la detección del AACI.

c. Comentarios respecto a las respuestas educativas extraordinarias y excepcionales.

d. Comentarios sobre las emociones y socialización en los AACI.

e. Comentarios respecto al AACI como persona, y no como grupo de individuos.

f. Comentarios que inciden en otros agentes.

- Comentarios que implican la labor del profesor, su formación y al sistema educativo en general:

- "Los centros educativos tienen ahora una ratio demasiado elevada y el comportamiento de los alumnos no es del todo bueno, es difícil hacer atenciones personalizadas. Estoy muy en desacuerdo con las medidas de sacar a los alumnos del grupo a trabajar con otro profesor, les suele señalar y se pierden un poco, además no hay personal especializado para trabajar con estos alumnos, no tienen suficientes conocimientos pues no son especialistas en las materias".

- "La implicación de muchos profesores es nula. Muchas veces creemos que un alumno de altas capacidades debe ser un "rarito" porque si no, no lo es.

- $\quad$ "El sistema educativo público español carece de posibilidades reales para atender a estos chavales ya que tiende a "tirar" de los de "abajo" para lograr unos niveles mínimos de exigencia que, si no se logran, se inventan. El sistema educativo falla desde la cantidad de horas lectivas en pupitre que se le imponen a un alumno a diario. Incidimos en materias y facetas que lo único que consiguen es rellenar horarios y permitir la jornada completa de los profesores. Y no existen recursos para motivar al estudiante con inquietudes, las capacidades vienen después y, si eso se cultiva, llega el pensamiento fructífero".

- "El profesorado de secundaria no está preparado para atender a este tipo de alumnado. A esto hay que añadir la falta de recursos tanto técnicos como humanos para hacerlo adecuadamente".

- "En la enseñanza pública tenemos tantos alumnos con dificultades que a los AACI no se les suele prestar atención, lo cual provoca en muchos casos fracaso escolar".

- "Sería conveniente tomarse más en serio este asunto y potenciar la formación del profesorado para poder atender convenientemente a estos alumnos y disponer de tiempo en el horario lectivo para su atención".

- "Todos los alumnos requieren atención específica; centrar el problema en los alumnos más dotados y en los menos dotados o en los que tienen buen rendimiento y en los que no lo tienen, deja al $65 \%$ del alumnado fuera. Una escuela inclusiva no puede permitírselo". 
- Comentarios respecto a la detección del AACI:

- "En mi opinión hay muchos más de los propiamente diagnosticados, y se prefiere tildar de problemáticos a niños antes de pensar que son AACI. No se hace ningún esfuerzo por detectarlos ni en primaria ni en Secundaria".

- "Pienso que no existen unos criterios diagnósticos compartidos y por lo tanto resulta peligroso diagnosticar a un alumno ya que nada va a ser igual desde ese momento. En algunos casos hemos podido observar y estudiar casos que han fracasado estrepitosamente al no cumplirse las expectativas del contexto y del propio alumno".

- "Creo que hoy en día existe un sobrediagnóstico de AACI, especialmente a edades tempranas. Tuve el caso de una alumna catalogada como AACI a los 8 años (yo imparto ESO), y cuando tenía 16 era una chica normal y corriente, para nada AACI".

- "El diagnóstico no es en absoluto determinante en la toma de decisiones educativas. Lo único prioritario es el alumno, sus características personales (como ser único e irrepetible), sus necesidades, motivaciones, gustos, condiciones emocionales, sociales... en pro de su bienestar integral y el desarrollo óptimo de sus capacidades, dentro del respeto máximo por su derecho a la infancia, la adolescencia y la felicidad".

- Comentarios respecto a las respuestas educativas extraordinarias y excepcionales:

- $\quad$ "En la experiencia que yo tengo con alumnos de altas capacidades, he comprobado lo positivo que es para ellos el adelantarlos un curso o dos cursos".

- "Soy partidario del enriquecimiento más que de la aceleración si puede afectar a su socialización".

- "Creo que las medidas tomadas con ello les hacen sentirse superiores a los demás y situarse siempre frente a los otros con una prepotencia que asusta".

- Comentarios sobre las emociones y socialización en los AACI:

- "Los AACI fallan sobre todo en el campo emocional, en las relaciones con los compañeros de clase. Es súper importante trabajar estos campos para que puedan integrarse y desarrollar todo su potencial. Son muy buenos en los que les gusta, pero pueden no hacer nada en las asignaturas que no les interesan. Imprescindible saber engancharlos".

- "Al AACI infantil o adolescente, como al resto del alumnado, hay que potenciar el máximo desarrollo de sus potencialidades, pero buscando y manteniendo siempre su equilibrio psicológico y emocional".

- "Considero que es fundamental atender a sus relaciones personales y las habilidades sociales, ya que muchos se detectan (y a veces diagnostican) por presentar dificultades en esos ámbitos de desarrollo".

- "Atender el estado emocional siempre es importante para todo tipo de alumnos".

- "Los AACI deberían integrarse lo más posible con su grupo de edad desarrollando a la vez sus capacidades excepcionales. El criterio fundamental es la integración plena en la sociedad, valorando sus virtudes y defectos como en el resto de las personas. Aislarlos convierte a los superdotados en un grupo de "mutantes" acechados por intereses ajenos a sus necesidades totales como personas". 
- Comentarios respecto al AACI como persona, y no como grupo de individuos.

- "Cada alumno-persona es un caso".

- "Creo que no es conveniente generalizar con AACI".

- "No todos los AACI presentan las mismas características, ni el mismo estado emocional y depende mucho del estímulo que tenga en casa y del nivel sociocultural de su familia".

- "No debemos perder de vista que son personas normales, algo peculiares, pero como todos,... hay mucha diversidad...".

- $\quad$ "Los AACI no se pueden valorar como si todos fuesen igual, debemos tener en cuenta sus características individuales para llevar a cabo una u otra medida".

- Comentarios que inciden en otros agentes:

- "Dar formación también a los padres que tienen un hijo AACI".

- "El apoyo y la percepción de sus compañeros de clase es imprescindible en la autovaloración que hacen los AACI".

- "Considero que un alumno con AACI presenta unas peculiaridades educativas que hacen que cada caso sea totalmente diferente de otros. Además considero de vital importancia el comportamiento de la familia y su colaboración”. 
